\title{
Dimensionality and Design of Isotropic Interactions that Stabilize Honeycomb, Square, Simple Cubic, and Diamond Lattices
}

\author{
Avni Jain \\ McKetta Department of Chemical Engineering, The University of Texas at Austin, \\ Austin, Texas 78712, USA \\ Jeffrey R. Errington \\ Department of Chemical and Biological Engineering, University of Buffalo, \\ The State University of New York, Buffalo, New York 14260-4200, USA \\ Thomas M. Truskett \\ McKetta Department of Chemical Engineering, The University of Texas at Austin, \\ Austin, Texas 78712, USA \\ (Received 25 May 2014; published 11 September 2014)
}

\begin{abstract}
We use inverse methods of statistical mechanics and computer simulations to investigate whether an isotropic interaction designed to stabilize a given two-dimensional lattice will also favor an analogous three-dimensional structure, and vice versa. Specifically, we determine the 3D-ordered lattices favored by isotropic potentials optimized to exhibit stable 2D honeycomb (or square) periodic structures, as well as the 2D-ordered structures favored by isotropic interactions designed to stabilize 3D diamond (or simple cubic) lattices. We find a remarkable "transferability" of isotropic potentials designed to stabilize analogous morphologies in 2D and 3D, irrespective of the exact interaction form, and we discuss the basis of this cross-dimensional behavior. Our results suggest that the discovery of interactions that drive assembly into certain 3D periodic structures of interest can be assisted by less computationally intensive optimizations targeting the analogous 2D lattices.
\end{abstract}

DOI: 10.1103/PhysRevX.4.031049

Material properties are intimately linked to structural characteristics featured at various length scales. Thus, discovering new ways to create materials with prescribed morphologies is a key challenge in their design for specific applications. In addition to the development of top-down material fabrication strategies, there has been considerable progress in bottom-up approaches in which the primary components (molecules, nanoparticles, colloids, etc.) are engineered to promote their self-assembly into targeted structures. Examples of the latter include assembly of lithographic masks [1], polymer membranes [2], magnetic nanostructures [3], and colloidal superlattices [4] for photonic materials $[5,6]$, to mention a few.

A critical part of any self-assembly design problem is understanding how tunable aspects of the interactions affect the thermodynamic stability of competing assembled states with different morphologies. For nanoscale to microscale

*truskett@che.utexas.edu

Published by the American Physical Society under the terms of the Creative Commons Attribution 3.0 License. Further distribution of this work must maintain attribution to the author(s) and the published article's title, journal citation, and DOI.
Subject Areas: Materials Science, Soft Matter, Statistical Physics

particles, this understanding has been guided in part via exploratory experiments and simulations to characterize the structures that spontaneously form from systems with various particle chemistries [7,8], shapes [9-15], and surface properties [16-19], as well as different dispersing solvents [20] and mixtures of assembling particles [21,22]. Highly coordinated lattices with, e.g., face-centered cubic or hexagonal symmetries in three dimensions [7] and triangular symmetry in two dimensions [23] are commonly observed in the experimental assembly of monodisperse particles with short-range, isotropic interactions. A broader array of thermodynamically stable 3D structures-including low-coordinated diamond and simple cubic (Sc) lattices of interest for technological applications $[24,25]$ - has also been demonstrated by computer simulations of monodisperse particles with softer, repulsive potentials [26-30], including those that model the interactions between elastic spheres [31] or star polymers [32]. Similar interactions favor open 2D structures as well, including honeycomb and square lattices [33-39] with, e.g., sterically stabilized magnetic particles in the presence of an external field [40] providing one novel experimental realization. Finally, low-coordinated lattices can also be stabilized by particles with patchy surfaces or faceted 
shapes, as demonstrated by experiments (mostly in 2D [41,42]) and simulations (in both 2D [43,44] and 3D $[16,18,45,46])$. For a given application, the choice of self-assembling components often hinges on practical considerations including the complexity and expense associated with particle synthesis and the kinetics of assembly.

Despite the fact that various interaction models are known to stabilize specific lattices of interest in a given spatial dimension (2D or 3D), much less is understood about how spatial dimension affects the design rules for assembly. For example, to what extent will an interaction designed to stabilize a given 2D lattice also favor an analogous 3D structure, and vice versa [47]? The answer is of fundamental interest and may also have important practical implications because finding interactions that stabilize lattices in $2 \mathrm{D}$ is a simpler and less computationally demanding material design problem than in 3D. Here, we study this question using computer simulations and model potentials designed by inverse statistical mechanical optimization $[48,49]$.

In particular, we determine the 3D-ordered lattices favored by models with isotropic potentials $\varphi_{\mathrm{hc}}\left(\varphi_{\mathrm{squ}}\right)$ optimized to exhibit stable 2D honeycomb (square) periodic structures, as well as the 2D-ordered structures favored by isotropic interactions $\varphi_{\text {dia }}\left(\varphi_{\mathrm{Sc}}\right)$ designed to stabilize $3 \mathrm{D}$ diamond (simple cubic) lattices [50]. As we show, the isotropic potentials optimized for either $2 \mathrm{D}$ or $3 \mathrm{D}$ target structures also do surprisingly well at stabilizing the analogous lattices in the other dimension.

A specified target lattice is the ground state for a given pair potential $\varphi$ and pressure $p$ if, and only if, it is mechanically stable at this condition and its zerotemperature chemical potential (i.e., molar enthalpy) is lower than that of all other mechanically stable competing structures. Here, we use a stochastic optimization approach (described in detail elsewhere [30]) to discover new model pairwise interactions $\varphi_{\text {target }}$ that maximize the range of density $\rho$ for which a 2D target lattice is the ground state. In our optimizations, we consider isotropic, convex-repulsive pair potentials that qualitatively mimic the soft, effective interactions of sterically stabilized colloids or nanoparticles [51]. The form we adopt can be expressed as [30]

$$
\begin{aligned}
\varphi(x)= & \epsilon\left\{A x^{-n}+\sum_{j=1}^{2} \lambda_{j}\left\{1-\tanh \left[k_{j}\left(x-\delta_{j}\right)\right]\right\}\right. \\
& \left.+f_{\text {shift }}(x)\right\} H\left[x_{\text {cut }}-x\right] .
\end{aligned}
$$

Here, $x=r / \sigma$ is a dimensionless interparticle separation, $\epsilon$ and $\sigma$ are characteristic energy and length scales, $x_{\text {cut }}$ is the dimensionless potential range, $H$ is the Heaviside step function, and $f_{\text {shift }}(x)=P x^{2}+Q x+R$ is a shifting function with fitting constants $P, Q, R$ chosen to ensure $\varphi\left(x_{\text {cut }}\right)=\varphi^{\prime}\left(x_{\text {cut }}\right)=\varphi^{\prime \prime}\left(x_{\text {cut }}\right)=0$. All together, there are nine dimensionless parameters that can be varied in the optimization algorithm ( $\left.x_{\text {cut }}, A, n, \lambda_{1}, k_{1}, \delta_{1}, \lambda_{2}, k_{2}, \delta_{2}\right)$; however, one is not independent of the others because we also require $\varphi(1) / \epsilon=1$. From here forward, we report quantities implicitly nondimensionalized by appropriate combinations of $\epsilon$ and $\sigma$.

To identify the ground-state phase diagram for a given pair potential $\varphi$, we compare the $p$-dependent, zero-temperature chemical potentials of a wide variety of Bravais and non-Bravais lattices in a "forward" calculation. Several methods for identifying candidate ground states are available, including evolutionary optimization $[52,53]$ and shape matching and machine learning algorithms [54]. In this study, we use simulated annealing optimization [30] to determine free-lattice parameters, which minimize the chemical potentials of the structures subject to the constraint of mechanical stability, as determined by phonon spectra analysis [55]. In 2D, the Bravais lattices consist of oblique, rhombic, square, rectangular, and triangular symmetries; here, we limit our consideration of non-Bravais lattices to honeycomb, kagome, and other five-vertex semiregular tilings, namely, snub-hexagonal, snub-square, and elongated-triangular. For 3D, we consider the following Bravais and non-Bravais lattices identified in a previous study on closely related model interactions [56]: fcc, bcc, $\mathrm{Sc}$, diamond, pyrochlore, body-centered orthogonal, hexagonal, rhombohedral, cI16, oC8, $\beta \mathrm{Sn}, \mathrm{A} 7, \mathrm{~A} 20$, and B10. While the methods employed both to determine the interaction potentials optimal for a target lattice and to compute the corresponding ground states are identical in 2D and 3D, we note that calculations are significantly faster in $2 \mathrm{D}$ than in $3 \mathrm{D}$ due to the smaller number of competing structures to consider in 2D and the reduced dimensionality of the lattice sum and the phonon spectra evaluations.

For computational efficiency of inverse optimizations in $2 \mathrm{D}$ or $3 \mathrm{D}$, only a limited set of competing structures can be considered for a specific target lattice, ideally consisting of the lattices that have the lowest chemical potentials for the interaction type over the density range of interest. Here, we use a simple iterative process for determining the competitive lattice pools. Specifically, we (1) begin with a trial set of competitive structures, (2) carry out an inverse optimization calculation using this competitive pool to obtain parameters for a trial optimal potential, (3) perform an extensive forward calculation to determine the groundstate phase diagram of the trial potential, and (4) as necessary, refine the competitive pool based on the lattices that appear in the forward calculation in step (3) and return to step (2). The final pools determined from this method contain a diverse array of structures in 2D and 3D [57].

To obtain information about the thermal stability of the target lattices, we also perform Monte Carlo quench simulations in which a high-temperature fluid is instantaneously cooled down to a much lower temperature to observe assembly of the target structure. Our simulation sizes are chosen such that larger systems do not affect the 


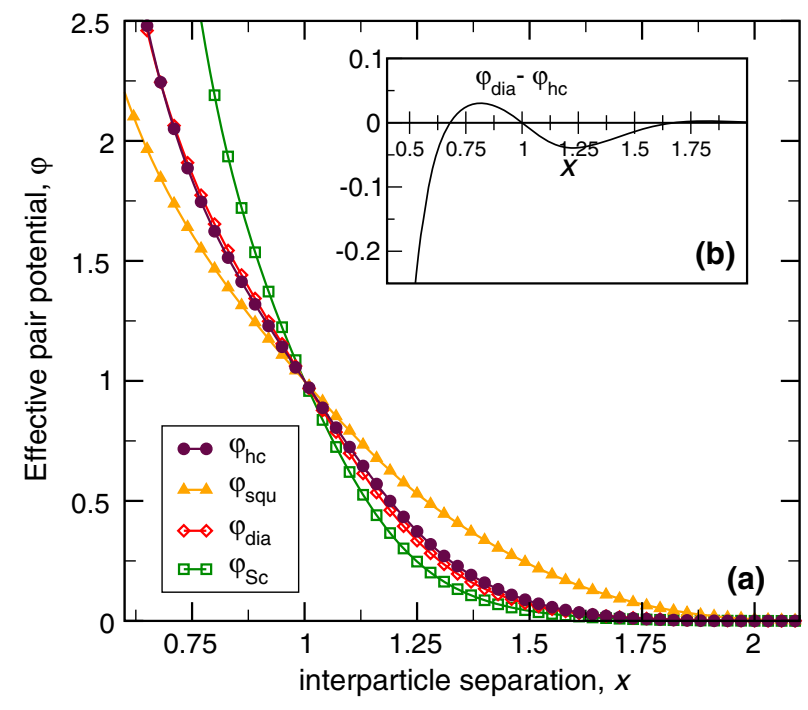

FIG. 1. Isotropic, convex-repulsive potentials, $\varphi_{\text {hc }}$ and $\varphi_{\text {squ }}$ (described in the text), which maximize the density range of mechanically stable 2D honeycomb-and square-lattice ground states, respectively. Also shown are previously designed potentials, $\varphi_{\mathrm{dia}}$ and $\varphi_{\mathrm{Sc}}$ [30], that maximize the density range of mechanically stable 3D diamond-and simple cubic-lattice ground states, respectively. The inset highlights subtle differences between $\varphi_{\text {dia }}$ and $\varphi_{\mathrm{hc}}$.

results (for more details, see Table $\mathrm{S} 1$ and discussion in the Supplemental Material [58]). We note that interactions previously optimized to stabilize 3D target ground states of diamond $\left(\varphi_{\text {dia }}\right)$ and simple cubic $\left(\varphi_{\mathrm{Sc}}\right)$ lattices over a wide range of density-using methods identical to those employed here-lead to target crystalline phases with good thermal stability [59].

The interaction potentials we obtain for maximizing the density range of 2D honeycomb- and square-lattice ground states [60] together with previously optimized interactions for diamond- and simple cubic-lattice ground states [30], are shown in Fig. 1. Notice that interactions $\varphi_{\mathrm{hc}}$ and $\varphi_{\mathrm{dia}}$ are remarkably similar to one another, despite the fact that they were obtained from optimizations favoring different (albeit analogous) structures in different spatial dimensions. As shown in the inset of Fig. 1, significant discrepancies between these potentials (i.e., the steeper repulsions of $\varphi_{\text {hc }}$ ) are present only for interparticle separations $x<0.6$ that, as we confirm below, are closer than the nearest-neighbor distance for the honeycomb or diamond lattices in the density range where the structures are stable for either model. Based on the similarity of these interactions, one might already expect that $\varphi_{\mathrm{hc}}$ and $\varphi_{\mathrm{dia}}$ would stabilize similar lattices in 2D and 3D. On the other hand, we see appreciable differences between the potentials $\varphi_{\text {squ }}$ and $\varphi_{\mathrm{Sc}}$ optimized to stabilize 2D square and 3D simple cubic lattices, respectively. Of the four interactions studied here, $\varphi_{\text {squ }}$ has the softest repulsive core and the longest range, while $\varphi_{\mathrm{Sc}}$ has the steepest core repulsion and the shortest range.

In Fig. 2, we show the results of our forward calculations, i.e., the $2 \mathrm{D}$ ground states for the four optimized potentials as a function of density [61]. Shaded regions represent densities where the ground state comprises two neighboring lattices in coexistence. First, we note that the 2D inverse optimization calculations succeed in their goal: stable honeycomb-and square-lattice ground states appear for $\varphi_{\mathrm{hc}}$ and $\varphi_{\mathrm{squ}}$, respectively, over very wide density ranges, especially when compared to those of other repulsive, isotropic interaction models $[36,39,62]$ known to form these phases. Perhaps more noticeable is not only that the $2 \mathrm{D}$ honeycomb lattice is stabilized over a similar density range by the $3 \mathrm{D}$-optimized $\varphi_{\text {dia }}$ (a result now expected based on the similarity to $\varphi_{\text {hc }}$ shown in Fig. 1), but also that the square lattice is stabilized over a wide density range by $\varphi_{\mathrm{Sc}}$ (despite significant differences compared to $\left.\varphi_{\text {squ }}\right)$. In other words, for both cases, stable $2 \mathrm{D}$
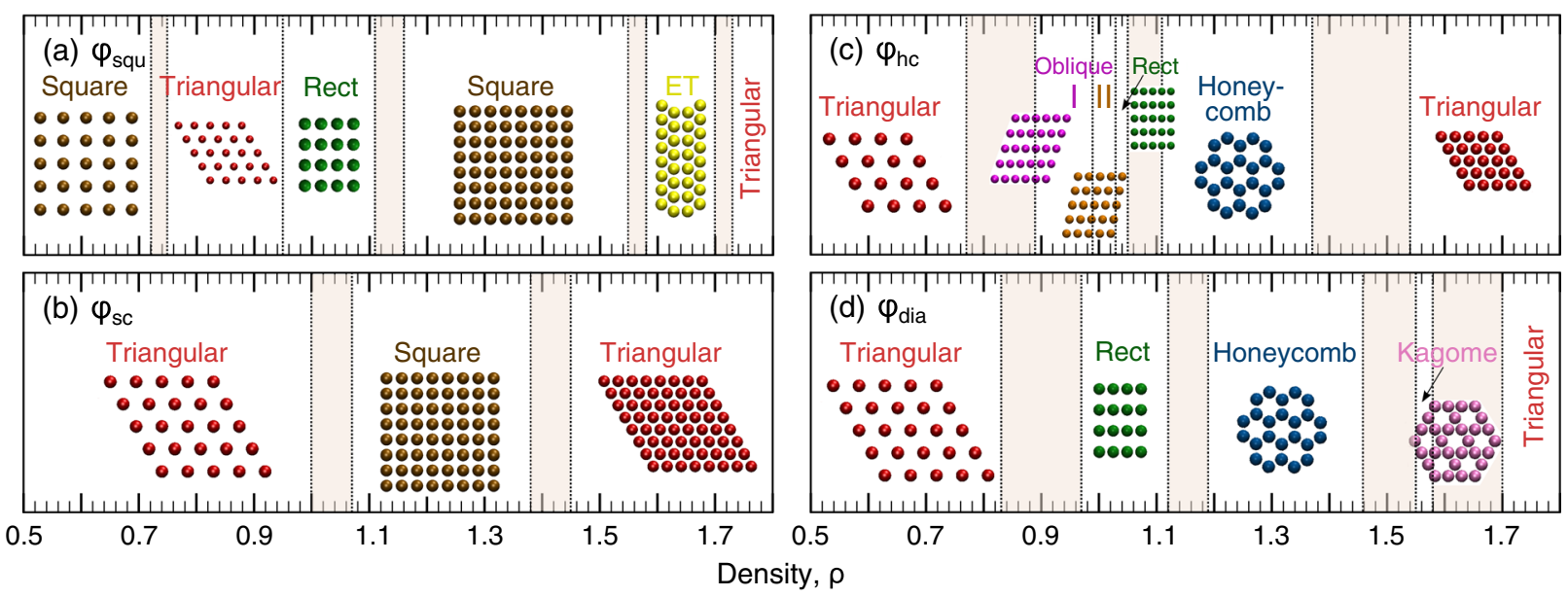

FIG. 2. $2 \mathrm{D}$ ground-state lattices as a function of density for (a) $\varphi_{\mathrm{squ}}$, (b) $\varphi_{\mathrm{Sc}}$, (c) $\varphi_{\mathrm{hc}}$, and (d) $\varphi_{\mathrm{dia}}$. Shaded regions represent coexistence between the neighboring lattices on the phase diagram. ET represents the elongated-triangle Archimedean tiling. Parameters for the oblique and rectangular (Rect) lattices are provided in Table S2 in the Supplemental Material [58]. 
ground states of interest are obtainable by optimizing interactions for a corresponding analogous target lattice in $3 \mathrm{D}$.

To test the same approach in the other direction, i.e., whether optimizing analogous 2D structures stabilizes 3D target lattices of interest, we also determine the 3D ground states for $\varphi_{\mathrm{hc}}$ and $\varphi_{\mathrm{squ}}$. The results, presented in Table I, show that $\varphi_{\mathrm{hc}}$ and $\varphi_{\text {squ }}$ indeed display wide stability regions for diamond-and simple cubic-lattice ground states, respectively. In fact, not only are the density ranges of the stable diamond lattice comparable for $\varphi_{\mathrm{hc}}$ and $\varphi_{\mathrm{dia}}$, but the density range of the simple cubic lattice for $\varphi_{\text {squ }}$ is even slightly wider than that of $\varphi_{\mathrm{Sc}}$ [63]. The latter result likely reflects the fact that the faster optimizations targeting 2D ground states enables a more thorough exploration of parameter space during the calculation than is practical in the $3 \mathrm{D}$ optimizations.

The fact that particles with isotropic interactions encoded to form 3D diamond (or simple cubic) lattices also display 2D honeycomb (or square) arrays, although nontrivial, is in some sense not surprising. The tetrahedrally coordinated diamond lattice itself consists of undulating interconnected trivalent honeycomb networks, and the simple cubic structure comprises square arrays stacked in registry. However, the outcome that particles with interactions

TABLE I. 3D ground states for $\varphi_{\mathrm{hc}}$ and $\varphi_{\text {squ }}$ with their corresponding density ranges and optimal lattice parameters. Roman numerals denote different structures of the same lattice type. Diamond and simple cubic ground-states are highlighted among other stable phases. Nomenclature is that of an earlier reference [56].

\begin{tabular}{lcl}
\hline \hline Lattice & Stability range & Lattice parameters \\
\hline Honeycomb-lattice forming potential, $\varphi_{\mathrm{hc}}$ \\
\hline bcc & {$[0.589,0.677]$} & \\
A7-I & {$[0.725,0.777]$} & $b / a: 2.23, u: 0.075$ \\
A7-II & {$[0.856,1.05]$} & $b / a: 4, u:[0.68,0.81]$ \\
Diamond & {$[\mathbf{1 . 0 9 1}, \mathbf{1 . 3 7 6}]$} & \\
Hexagonal & {$[1.468,1.474]$} & $c / a: 1.38$ \\
A20-I & {$[1.477,1.498]$} & $b / a: 1.72, c / a: 2.8, y: 0.5$ \\
A20-II & {$[1.54,1.851]$} & $b / a: 1.8, c / a: 0.65, y: 0.66$ \\
Square-lattice & forming potential, $\varphi_{\mathrm{squ}}$ \\
\hline$\beta$ Sn-I & {$[0.587,0.641]$} & $c / a: 2.67$ \\
B10-I & {$[0.664,0.798]$} & $c / a: 0.4, z: 0.5$ \\
fcc & {$[0.828,0.961]$} & \\
A20-I & {$[0.991,1.047]$} & $b / a: 1.0, c / a: 0.68, y: 0.8$ \\
oC8-Ga & {$[1.056,1.094]$} & $b / a: 1.0, c / a: 1.5$ \\
& & $u: 0.75, v: 0.163$ \\
B10-II & {$[1.1,1.267]$} & $c / a:[0.72,0.73]$, \\
& & $z:[0.38,0.39]$ \\
A20-II & {$[1.282,1.298]$} & $b / a: 2.29, c / a: 1.79, y: 0.08$ \\
$\beta$ Sn-II & {$[1.322,1.478]$} & $c / a:[0.57,0.64]$ \\
Hexagonal-I & {$[1.49,1.592]$} & $c / a:[0.887,0.9]$ \\
Simple cubic & {$[\mathbf{1 . 6 0 6}, \mathbf{1 . 9 4 9}]$} & \\
$\beta$ Sn-III & {$[1.98,2.106]$} & $c / a: 2.74$ \\
\hline \hline
\end{tabular}

designed to stabilize 2D honeycomb (or square) lattices also favor diamond (or simple cubic) lattices and not other morphologies containing honeycomb (or square) motifs such as graphite (or body-centered cubic) structures is much more interesting.

To understand these results, it is helpful to recall thatfor isotropic potentials-the zero-temperature chemical potential depends only on the pair interaction and properties of coordination shells located at distances closer than the interaction cutoff, $x<x_{\text {cut }}$. In Fig. 3, we plot the interparticle separations corresponding to the first, second, and third coordination shells $\left\{x_{1}, x_{2}, x_{3}\right\}$ for the four lattices of interest here-honeycomb, square, diamond, and simple cubic - considering densities where these lattices are the ground states for the models $\varphi_{\mathrm{hc}}$ and $\varphi_{\mathrm{squ}}$. First, note that there is considerable overlap between the coordination-shell distances of the honeycomb and diamond structures. Thus, an isotropic potential that stabilizes a honeycomb structure in 2D is expected to be an excellent (if not necessarily optimal) candidate for forming a diamond lattice in $3 \mathrm{D}$, and vice versa. This helps to explain the near identical potentials, $\varphi_{\mathrm{hc}}$ and $\varphi_{\text {dia }}$, despite their being obtained via optimization of different target structures in different spatial dimensions.

To gain further insight, we also compare the coordination-shell distances of the honeycomb lattice with another related 3D structure, graphite, which consists of

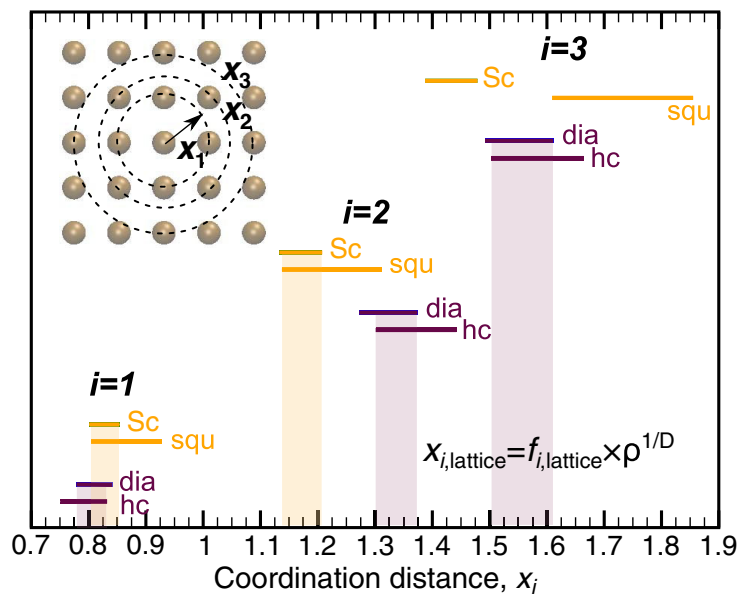

FIG. 3. Separations corresponding to first $(i=1)$, second $(i=2)$, and third $(i=3)$ coordination shells within the stability range of honeycomb (hc) $\rho=[1.11,1.37]$ and diamond (dia) $\rho=$ $[1.09,1.38]$ ground states for $\varphi_{\mathrm{hc}}$, as well as square (squ) $\rho=$ $[1.16,1.55]$ and simple cubic $(\mathrm{Sc}) \rho=[1.61,1.95]$ ground states for $\varphi_{\text {squ }}$. The coordination distance $\left(x_{i}\right)$ for shell $i$ is related to density $(\rho)$ by $x_{i, \text { lattice }}=f_{i \text {, lattice }} \times \rho^{1 / \mathrm{D}}$, where $D=2$ for hc and squ, and $D=3$ for dia and Sc. For hc, $f_{1}=0.877383$, $f_{2}=\sqrt{3} f_{1}, f_{3}=2 f_{1}$; for squ, $f_{1}=1, f_{2}=\sqrt{2} f_{1}, f_{3}=2 f_{1}$; for dia, $f_{1}=0.866025, f_{2}=\sqrt{8 / 3} f_{1}, f_{3}=\sqrt{11 / 3} f_{1}$; for Sc, $f_{1}=1, f_{2}=\sqrt{2} f_{1}, f_{3}=\sqrt{3} f_{1}$. Shaded regions indicate overlap of coordination-shell distances for analogous 2D and 3D stable lattice ground states. 
stacks of 2D honeycomb (i.e., graphene) sheets. Note that only the nearest-neighbor distances of mechanically stable 3D graphite lattices align with the first coordination-shell separations of 2D honeycomb structures, and there is substantial mismatch of other relevant coordination distances $(i \geq 2)$ (see Fig. S1 in the Supplemental Material [58]). In this important sense, graphite-while closely related to the honeycomb lattice in other ways-is not as analogous to honeycomb as the $3 \mathrm{D}$ diamond structure is in its relation between interaction and coordination-shell structure, and is thus not favored as a ground state by $\varphi_{\mathrm{hc}}$ at any density. In comparing the other case of square versus simple cubic lattices, we see that the first two coordination shells of these structures similarly overlap, but the third shell positions are not in alignment. This result— together with the ground-state calculations presented abovesuggests that, for short-range interactions, the common separation distances between the nearest-and next-nearest neighbors for square and simple cubic structures is enough to allow for an optimal 2D square-forming potential to assemble into 3D simple cubic structures, and vice versa. However, the differences in the third-shell distances might help to explain the significant variations in the optimized potentials targeting $2 \mathrm{D}$ square $\left(\varphi_{\text {squ }}\right)$ versus $3 \mathrm{D}$ simple cubic $\left(\varphi_{\mathrm{Sc}}\right)$ lattices shown in Fig. 1.

In Figs. 4(a)-4(d), we present snapshots of configurations obtained from the Monte Carlo quench simulations for the four potential models. Configurations for the 3D diamond and simple cubic lattice obtained via quenching systems interacting with $2 \mathrm{D}$-optimized $\varphi_{\mathrm{hc}}$ and $\varphi_{\text {squ }}$ interactions are shown in Figs. 4(e) and 4(f), respectively. The structures obtained are inspected visually, and their configurational energies and pair distribution functions $g(r)$ are compared to equilibrated lattice structures at the corresponding densities and temperatures (see Table S1 in the Supplemental Material [58]). In Fig. 4(g), the complete overlap of the pair distribution functions of the quenched fluid (red circles) and the equilibrated simple cubic structure (black dashed lines) demonstrates the assembly of a defect-free simple cubic crystal. The $\varphi_{\mathrm{hc}}$ model similarly assembles into a (slightly defective) diamond structure as illustrated by the comparison of the pair distribution functions in Fig. 4(h). The energy of the quenched configuration is only $0.09 \%$ higher than the perfectly equilibrated diamond lattice. Nonetheless, in all cases, the structures obtained by the Monte Carlo quench procedure match the expectations of the ground-state calculations.

In summary, we investigate the cross-dimensional phase behavior of specifically designed isotropic interactions with low coordination. In particular, we determine the 3D-ordered lattices favored by isotropic potentials $\varphi_{\mathrm{hc}}$ $\left(\varphi_{\text {squ }}\right)$ optimized to exhibit stable 2D honeycomb (square) lattice structures, as well as the $2 \mathrm{D}$ periodic structures favored by isotropic potentials $\varphi_{\mathrm{dia}}\left(\varphi_{\mathrm{Sc}}\right)$ optimized to assemble into 3D diamond (simple cubic) morphologies.

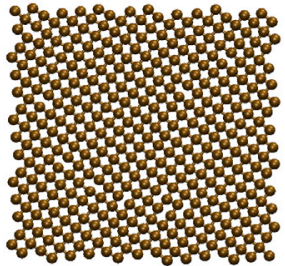

(a) square from $\varphi_{\mathrm{Sc}}$ $T=0.03, \rho \sigma^{2}=1.23$

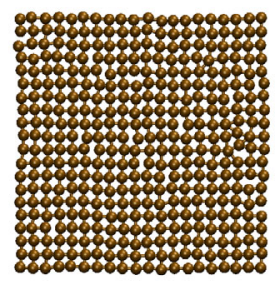

(c) square from $\varphi_{\text {squ }}$ $T=0.02, \rho \sigma^{2}=1.35$

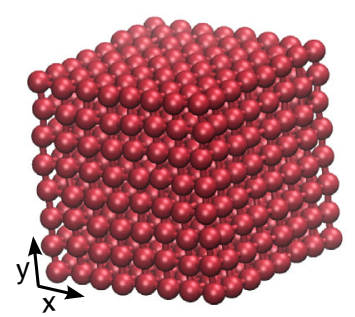

(e) simple cubic from $\varphi_{\text {squ }}$ $T=0.01, \rho \sigma^{3}=1.77$

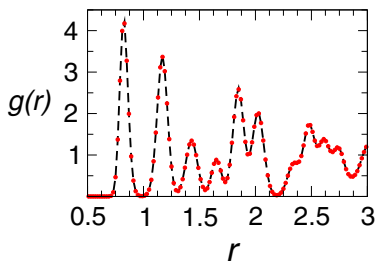

(g) Comparing $g(r)$ for $\varphi_{\text {squ }}$ $T=0.01, \rho \sigma^{3}=1.77$

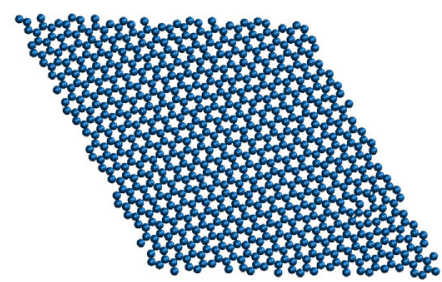

(b) honeycomb from $\varphi_{\text {dia }}$ $T=0.03, \rho \sigma^{2}=1.32$

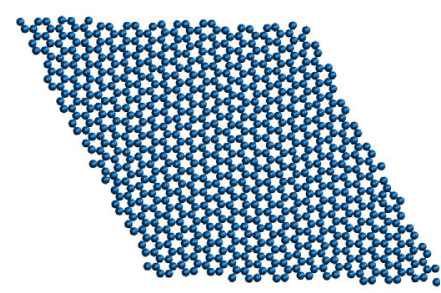

(d) honeycomb from $\varphi_{\mathrm{hc}}$ $T=0.02, \rho \sigma^{2}=1.23$

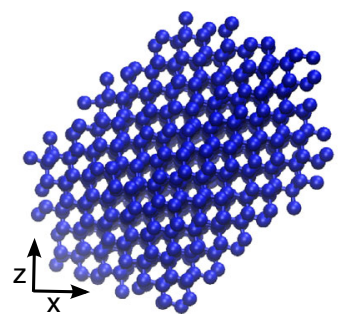

(f) diamond from $\varphi_{\mathrm{hc}}$ $T=0.025, \rho \sigma^{3}=1.23$

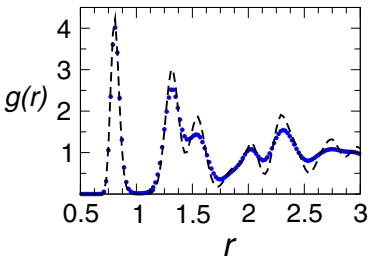

(h) Comparing $g(r)$ for $\varphi_{\text {hc }}$ $T=0.025, \rho \sigma^{3}=1.23$
FIG. 4. Snapshots of 2D configurations obtained from Monte Carlo quenches of (a) $\varphi_{\mathrm{Sc}}$, (b) $\varphi_{\text {dia }}$, (c) $\varphi_{\text {squ }}$, (d) $\varphi_{\mathrm{hc}}$ and $3 \mathrm{D}$ configurations obtained from quenches of (e) $\varphi_{\text {squ }}$ and (f) $\varphi_{\mathrm{hc}}$. For the $3 \mathrm{D}$ structures, we plot the corresponding pair distribution functions $g(r)$ of the quenched configurations (dots) and the perfectly equilibrated lattice structures (dashed lines) in $(\mathrm{g})$ and $(\mathrm{h})$, at the specific temperature $(T)$ and density $(\rho)$, to demonstrate the structural similarities. The comparison between the pair distribution functions of the annealed and perfectly equilibrated diamond lattice in (h) shows that the annealed structure is not quite defect free, but the configuration energy is only $0.09 \%$ higher than that of the perfect lattice. For more details, see Table S1 in the Supplemental Material [58].

We find surprising transferability of interactions designed to stabilize analogous structures in $2 \mathrm{D}$ and $3 \mathrm{D}$, and we gain insight into this behavior by studying the different ways in which information in the analogous target structures encodes itself in the optimal isotropic potentials through coordination-shell geometry. 
One practical implication of the observed physics in this study is that the design of certain 3D lattices can greatly benefit from knowledge of potentials derived to maximize the stability of analogous 2D structures, information that can be obtained at relatively modest computational expense. The computational efficiency gained from this approach might be most valuable in multistep optimization processes, where the goal to search for an interaction potential favoring a target structure is only one of several objectives within the design calculation. It will also be interesting in future studies to explore the effects of the interaction range on the cross-dimensional behavior of isotropic interactions obtained through inverse design, especially where one limits the potential range to encompass only two coordination shells. While we focus here on the dimensionality dependence of design rules pertaining to target structures formed by isotropic interactions, it will also be informative to study the effect of spatial dimension on other classes of interactions, e.g., short-ranged anisotropic interactions of patchy particles relevant to $2 \mathrm{D}$ and $3 \mathrm{D}$ assembly scenarios.

Finally, in the context of cross-dimensional freezing behavior, we note the differences between the soft repulsive interactions studied here-which enthalpically stabilize low-coordinated periodic structures-and hard-sphere systems where entropy drives the particles to adopt closepacked periodic structures at high density. For the latter, crystallization from the fluid becomes increasingly more challenging in higher spatial dimensions due to correspondingly stronger geometric frustration $[64,65]$. The role that frustration plays in the dimensionality dependence of crystallization for particles with considerably softer repulsions remains a potentially rich area for future study.

T. M. T. acknowledges support from the Welch Foundation (F-1696) and the National Science Foundation (CBET-1403768). J. R. E. acknowledges support from the National Science Foundation (CHE1012356). We also acknowledge the Texas Advanced Computing Center (TACC) at The University of Texas at Austin for providing HPC resources that have contributed to the research results reported within this paper.

[1] J. Zhang, Y. Li, X. Zhang, and B. Yang, Colloidal SelfAssembly Meets Nanofabrication: From Two-Dimensional Colloidal Crystals to Nanostructure Arrays, Adv. Mater. 22, 4249 (2010).

[2] D. Wu, F. Xu, B. Sun, R. Fu, H. He, and K. Matyjaszewski, Design and Preparation of Porous Polymers, Chem. Rev. 112, 3959 (2012).

[3] C. C. Wang, A. O. Adeyeye, and N. Singh, Magnetic Antidot Nanostructures: Effect of Lattice Geometry, Nanotechnology 17, 1629 (2006).
[4] Y. Xia, B. Gates, Y. Yin, and Y. Lu, Monodispersed Colloidal Spheres: Old Materials with New Applications, Adv. Mater. 12, 693 (2000).

[5] S. Furumi, H. Fudouzi, and T. Sawada, Self-Organized Colloidal Crystals for Photonics and Laser Applications, Laser Photonics Rev. 4, 205 (2010).

[6] G. von Freymann, V. Kitaev, B. V. Lotschz, and G. A. Ozin, Bottom-Up Assembly of Photonic Crystals, Chem. Soc. Rev. 42, 2528 (2013).

[7] C. B. Murray, C. R. Kagan, and M. G. Bawendi, Synthesis and Characterization of Monodisperse Nanocrystals and Close-Packed Nanocrystal Assemblies, Annu. Rev. Mater. Sci. 30, 545 (2000).

[8] J. Young Kim, O. Voznyy, D. Zhitomirsky, and E. H. Sargent, 25th Anniversary Article: Colloidal Quantum Dot Materials and Devices: A Quarter-Century of Advances, Adv. Mater. 25, 4986 (2013).

[9] S. C. Glotzer and M. J. Solomon, Anisotropy of Building Blocks and Their Assembly into Complex Structures, Nat. Mater. 6, 557 (2007).

[10] Y. Xia, Y. Xiong, B. Lim, and S. E. Skrabalak, Shape-Controlled Synthesis of Metal Nanocrystals: Simple Chemistry Meets complex Physics?, Angew. Chem., Int. Ed. Engl. 48, 60 (2009).

[11] P. F. Damasceno, M. Engel, and S. C. Glotzer, Predictive Self-Assembly of Polyhedra into Complex Structures, Science 337, 453 (2012).

[12] U. Agarwal and F. A. Escobedo, Mesophase Behaviour of Polyhedral Particles, Nat. Mater. 10, 230 (2011).

[13] J. Henzie, M. Gruenwald, A. Widmer-Cooper, P. L. Geissler, and P. Yang, Self-Assembly of Uniform Polyhedral Silver Nanocrystals into Densest Packings and Exotic Superlattices, Nat. Mater. 11, 131 (2012).

[14] Z. Quan, H. Xu, C. Wang, X. Wen, Y. Wang, J. Zhu, R. Li, C. J. Sheehan, Z. Wang, D.-M. Smilgies, Z. Luo, and J. Fang, Solvent-Mediated Self-Assembly of Nanocube Superlattices, J. Am. Chem. Soc. 136, 1352 (2014).

[15] S. Sacanna, D. J. Pine, and G.-R. Yi, Engineering Shape: The Novel Geometries of Colloidal Self-Assembly, Soft Matter 9, 8096 (2013).

[16] C. Vega and P. A. Monson, Solid-Fluid Equilibrium for a Molecular Model with Short Ranged Directional Forces, J. Chem. Phys. 109, 9938 (1998).

[17] D. J. Kraft, R. Ni, F. Smallenburg, M. Hermes, K. Yoon, D. A. Weitz, A. van Blaaderen, J. Groenewold, M. Dijkstra, and W. K. Kegel, Surface Roughness Directed SelfAssembly of Patchy Particles into Colloidal Micelles, Proc. Natl. Acad. Sci. U.S.A. 109, 10787 (2012).

[18] G.-R. Yi, D. J. Pine, and S. Sacanna, Recent Progress on Patchy Colloids and Their Self-Assembly, J. Phys. Condens. Matter 25, 193101 (2013).

[19] A. Jayaraman, Polymer Grafted Nanoparticles: Effect of Chemical and Physical Heterogeneity in Polymer Grafts on Particle Assembly and Dispersion, J. Polym. Sci., Part B Polym. Phys. 51, 524 (2013).

[20] A. Courty, J. Richardi, P.-A. Albouy, and M.-P. Pileni, How to Control the Crystalline Structure of Supracrystals of 5-nm Silver Nanocrystals, Chem. Mater. 23, 4186 (2011).

[21] E. V. Shevchenko, D. V. Talapin, N. A. Kotov, S. O’Brien, and C. B. Murray, Structural Diversity in Binary 
Nanoparticle Superlattices, Nature (London) 439, 55 (2006).

[22] M. J. Pavan, E. Ploshnik, and R. Shenhar, Nanoparticle Assembly on Topographical Polymer Templates: Effects of Spin Rate, Nanoparticle Size, Ligand, and Concentration, J. Phys. Chem. B 116, 13922 (2012).

[23] S. Kinge, M. Crego-Calama, and D. N. Reinhoudt, SelfAssembling Nanoparticles at Surfaces and Interfaces, ChemPhysChem 9, 20 (2008).

[24] K. M. Ho, C. T. Chan, and C. M. Soukoulis, Existence of a Photonic Gap in Periodic Dielectric Structures, Phys. Rev. Lett. 65, 3152 (1990).

[25] H. Sami Sözüer and J. W. Haus, Photonic Bands: SimpleCubic Lattice, J. Opt. Soc. Am. B 10, 296 (1993).

[26] T. Yoshida and S. Kamakura, Theory of Melting at High Pressures. II, Prog. Theor. Phys. 47, 1801 (1972).

[27] Yu. D. Fomin, N. V. Gribova, V. N. Ryzhov, S. M. Stishov, and D. Frenkel, Quasibinary Amorphous Phase in a ThreeDimensional System of Particles with Repulsive-Shoulder Interactions, J. Chem. Phys. 129, 064512 (2008).

[28] É. Marcotte, F. H. Stillinger, and S. Torquato, Communication: Designed Diamond Ground State via Optimized Isotropic Monotonic Pair Potentials, J. Chem. Phys. 138, 061101 (2013).

[29] E. Edlund, O. Lindgren, and M. Nilsson Jacobi, Using the Uncertainty Principle to Design Simple Interactions for Targeted Self-Assembly, J. Chem. Phys. 139, 024107 (2013).

[30] A. Jain, J. R. Errington, and T. M. Truskett, Inverse Design of Simple Pairwise Interactions with Low-Coordinated 3D Lattice Ground States, Soft Matter 9, 3866 (2013).

[31] J. C. Pámies, A. Cacciuto, and D. Frenkel, Phase Diagram of Hertzian Spheres, J. Chem. Phys. 131, 044514 (2009).

[32] M. Watzlawek, C. N. Likos, and H. Löwen, Phase Diagram of Star Polymer Solutions, Phys. Rev. Lett. 82, 5289 (1999).

[33] E. A. Jagla, Minimum Energy Configurations of Repelling Particles in Two Dimensions, J. Chem. Phys. 110, 451 (1999).

[34] P. J. Camp, Structure and Phase Behavior of a TwoDimensional System with Core-Softened and Long-Range Repulsive Interactions, Phys. Rev. E 68, 061506 (2003).

[35] G. Malescio and G. Pellicane, Stripe Phases from Isotropic Repulsive Interactions, Nat. Mater. 2, 97 (2003).

[36] É. Marcotte, F. H. Stillinger, and S. Torquato, Unusual Ground States via Monotonic Convex Pair Potentials, J. Chem. Phys. 134, 164105 (2011).

[37] G. Zhang, F. H. Stillinger, and S. Torquato, Probing the Limitations of Isotropic Pair Potentials to Produce GroundState Structural Extremes via Inverse Statistical Mechanics, Phys. Rev. E 88, 042309 (2013).

[38] T. Lafitte, S. K. Kumar, and A.Z. Panagiotopoulos, SelfAssembly of Polymer-Grafted Nanoparticles in Thin Films, Soft Matter 10, 786 (2014).

[39] D. E. Dudalov, Yu. D. Fomin, E. N. Tsiok, and V. N. Ryzhov, Anomalous Melting Scenario of the TwoDimensional Core-Softened System, Phys. Rev. Lett. 112, 157803 (2014).

[40] N. Osterman, D. Babič, I. Poberaj, J. Dobnikar, and P. Ziherl, Observation of Condensed Phases of Quasiplanar Core-Softened Colloids, Phys. Rev. Lett. 99, 248301 (2007).
[41] Q. Chen, S. Chul Bae, and S. Granick, Directed SelfAssembly of a Colloidal Kagome Lattice, Nature (London) 469, 381 (2011).

[42] W. H. Evers, B. Goris, S. Bals, M. Casavola, J. de Graaf, R. van Roij, M. Dijkstra, and D. Vanmaekelbergh, LowDimensional Semiconductor Superlattices Formed by Geometric Control over Nanocrystal Attachment, Nano Lett. 13, 2317 (2013).

[43] M. Antlanger, G. Doppelbauer, and G. Kahl, On the Stability of Archimedean Tilings Formed by Patchy Particles, J. Phys. Condens. Matter 23, 404206 (2011).

[44] J. A. Millan, D. Ortiz, G. van Anders, and S. C. Glotzer, Self-Assembly of Archimedean Tilings with Enthalpically and Entropically Patchy Polygons, ACS Nano 8, 2918 (2014).

[45] E. Bianchi, R. Blaak, and C. N. Likos, Patchy Colloids: State of the Art and Perspectives, Phys. Chem. Chem. Phys. 13, 6397 (2011).

[46] F. Romano and F. Sciortino, Patterning Symmetry in the Rational Design of Colloidal Crystals, Nat. Commun. 3, 975 (2012).

[47] The term "analogous structures" refers to the pairs of 2D-3D lattices (e.g., honeycomb-diamond and square-simple cubic) that have specific coordination-shell similarities which allows a single isotropic pair potential to favor the stability of both lattices. This structural similarity is addressed in the discussion of Fig. 3 and in Fig. S1 (in the Supplemental Material [58]).

[48] S. Torquato, Inverse Optimization Techniques for Targeted Self-Assembly, Soft Matter 5, 1157 (2009).

[49] A. Jain, J. A. Bollinger, and T. M. Truskett, Perspective: Inverse Methods for Material Design, AIChE J. 60, 2732 (2014).

[50] We note that particles confined to a 2D monolayer, such as at a liquid-liquid interface or on a substrate, may interact via an effective pair potential that is different from the one that the same particles experience in a 3D bulk fluid.

[51] P. Schapotschnikow, R. Pool, and T. J. H. Vlugt, Molecular Simulations of Interacting Nanocrystals, Nano Lett. 8, 2930 (2008).

[52] D. Gottwald, G. Kahl, and C. N. Likos, Predicting Equilibrium Structures in Freezing Processes, J. Chem. Phys. 122, 204503 (2005).

[53] E. Bianchi, G. Doppelbauer, L. Filion, M. Dijkstra, and G. Kahl, Predicting Patchy Particle Crystals: Variable Box Shape Simulations and Evolutionary Algorithms, J. Chem. Phys. 136, 214102 (2012).

[54] C. L. Phillips and G. A. Voth, Discovering Crystals Using Shape Matching and Machine Learning, Soft Matter 9, 8552 (2013).

[55] N. W. Ashcroft and N. D. Mermin, Solid State Physics (Saunders College, Philadelphia, 1976).

[56] S. Prestipino, F. Saija, and G. Malescio, The ZeroTemperature Phase Diagram of Soft-Repulsive Particle Fluids, Soft Matter 5, 2795 (2009).

[57] Using the approach outlined in the text, the competitive pools used in optimizations targeting the honeycomb lattice consisted of triangular, square, kagome, snub-square, elongated-triangular, rectangular $(b / a=1.49)$, rectangular $(b / a=1.54)$, rectangular $\quad(b / a=1.56)$, rectangular 
$(b / a=1.7)$, and snub-hexagonal lattices. For the square lattice, the final pool comprised triangular, oblique $(b / a=1.514, \theta=1.234)$, kagome, honeycomb, elongated-triangular, snub-hexagonal, and snub-square lattices. For diamond, the pool [30] consisted of fcc, WUR, SH $(c / a=1.5), \beta \mathrm{Sn}(c / a=1.39), \beta \mathrm{Sn}(c / a=1.25), \mathrm{A} 7$ $(b / a=3.79, u=0.1385), \quad$ and $\mathrm{A} 20 \quad(b / a=1.728$, $c / a=0.626, y=0.167)$ lattices. For simple cubic, the pool [30] comprised fcc, bcc, diamond, Hexagonal (SH) $(c / a=1), \mathrm{SH}(c / a=1.08), \mathrm{SH}(c / a=1.172), \mathrm{A} 20$ $(b / a=1.72, c / a=0.66, y=0.67), \beta \operatorname{Sn}(c / a=0.873)$, $\beta \mathrm{Sn}(c / a=0.78)$, and $\beta \mathrm{Sn}(c / a=1.75)$ lattices. Here, $b / a$ and $c / a$ denote the aspect ratio of the sides of the unit cell, and $\theta$ is the angle between the two sides. The other symbols $u$ and $y$, we adopt here, are the same as those used in a previous study [56].

[58] See Supplemenatl Material at http://link.aps.org/ supplemental/10.1103/PhysRevX.4.031049 for details on the Monte Carlo isochoric quenching simulations, 2D ground-state phase diagrams for all the four optimal potentials, and coordination shell analysis corresponding to the honeycomb-forming potential for honeycomb, diamond and graphite structures.

[59] A. Jain, J. R. Errington, and T. M. Truskett, Communication: Phase Behavior of Materials with Isotropic Interactions Designed by Inverse Strategies to Favor Diamond and Simple Cubic Lattice Ground States, J. Chem. Phys. 139, 141102 (2013).

[60] Optimal parameters for the honeycomb-forming interaction $\varphi_{\mathrm{hc}} \quad$ are $A=0.326914, \quad n=3.63306, \quad \lambda_{1}=0.286436$, $k_{1}=3.6569, \delta_{1}=1.26977, \lambda_{2}=1.03258, k_{2}=3.03683$, $\delta_{2}=1.1557, \quad P=-0.175071, \quad Q=0.800825, \quad R=$ -0.937142 , and $x_{\text {cut }}=2.03291$. For the square-forming potential $\varphi_{\text {squ }}$, they are $A=0.0946889, n=3.53953$, $\lambda_{1}=0.32989, \quad k_{1}=1.89197, \quad \delta_{1}=1.99003, \quad \lambda_{2}=$ $0.062012, \quad k_{2}=5.89983, \quad \delta_{2}=1.0809, \quad P=0.433908$, $Q=-2.4391, R=3.46552$, and $x_{\text {cut }}=2.27813$.

[61] Also see Table S2 in the Supplemental Material [58], which tabulates the stable ground-state lattices with their corresponding density ranges and lattice parameters

[62] E. Marcotte, F. H. Stillinger, and S. Torquato, Optimized Monotonic Convex Pair Potentials Stabilize LowCoordinated Crystals, Soft Matter 7, 2332 (2011).

[63] Ground states and finite-temperature phase boundaries for $\varphi_{\text {dia }}$ and $\varphi_{\mathrm{Sc}}$ have been determined previously and are presented in detail elsewhere [30,59].

[64] J. A. van Meel, D. Frenkel, and P. Charbonneau, Geometrical Frustration: A Study of Four-Dimensional Hard Spheres, Phys. Rev. E 79, 030201 (2009).

[65] J. A. van Meel, B. Charbonneau, A. Fortini, and P. Charbonneau, Hard-Sphere Crystallization Gets Rarer with Increasing Dimension, Phys. Rev. E 80, 061110 (2009). 